\title{
CONVEXITY ESTIMATES FOR NONLINEAR ELLIPTIC EQUATIONS AND APPLICATION TO FREE BOUNDARY PROBLEMS
}

\section{ESTIMATIONS DE CONVEXITÉ POUR DES ÉQUATIONS NON-LINÉAIRES ELLIPTIQUES ET APPLICATION À DES PROBLÈMES DE FRONTIÈRE LIBRE}

\author{
Jean DOLBEAULT ${ }^{\mathrm{a}}$, Régis MONNEAU ${ }^{\mathrm{b}}$ \\ ${ }^{a}$ CEREMADE, U.M.R. C.N.R.S. no. 7534, Univ. Paris IX-Dauphine, pl. de Lattre de Tassigny, \\ 75775 Paris cedex 16, France \\ ${ }^{\mathrm{b}}$ CERMICS, E.N.P.C., 6 \& 8 avenue Blaise Pascal, cité Descartes, Champs-sur-Marne, \\ 77455 Marne-La-Vallée, France
}

Received 17 January 2001

ABSTRACT. - We prove the convexity of the set which is delimited by the free boundary corresponding to a quasi-linear elliptic equation in a 2-dimensional convex domain. The method relies on the study of the curvature of the level lines at the points which realize the maximum of the normal derivative at a given level, for analytic solutions of fully nonlinear elliptic equations. The method also provides an estimate of the gradient in terms of the minimum of the (signed) curvature of the boundary of the domain, which is not necessarily assumed to be convex. (C) 2002 L'Association Publications de l'Institut Henri Poincaré. Published by Elsevier B.V. All rights reserved

\section{AMS classification: $35 \mathrm{~J} 25 ; 35 \mathrm{~J} 67 ; 35 \mathrm{R} 35$}

Keywords: Quasi-linear elliptic equations; Fully nonlinear elliptic equations; Free boundary; Gradient estimates; Fréchet formula; Curvature of level sets; Obstacle problem; Coincidence set

RÉSUMÉ. - Nous démontrons la convexité de l'ensemble délimité par la frontière libre correspondant à une équation quasi-linéaire elliptique définie sur un domaine convexe en dimension 2. La méthode repose sur l'étude de la courbure des lignes de niveau aux points qui réalisent le maximum de la dérivée normale pour un niveau donné, pour des solutions analytiques d'équations elliptiques complètement non linéaires. La méthode donne aussi une estimation du gradient en fonction du minimum de la courbure (signée) du bord du domaine, qui n'est pas nécessairement supposé convexe.

(C) 2002 L'Association Publications de l'Institut Henri Poincaré. Published by Elsevier B.V. All rights reserved

E-mail addresses: dolbeaul@ceremade.dauphine.fr (J. Dolbeault), monneau@ cermics.enpc.fr (R. Monneau). 


\section{Introduction and main results}

Consider a solution of the following free boundary problem

$$
\begin{aligned}
& \operatorname{div}\left(a\left(|\nabla u|^{2}\right) \nabla u\right)=f(u) \quad \text { in } \Omega \backslash \Lambda, \\
& 0=\left.u\right|_{\partial \Lambda}<u<\left.u\right|_{\partial \Omega}=u_{0} \quad \text { in } \Omega \backslash \Lambda, \\
& \partial_{n} u=0 \quad \text { on } \partial \Lambda,
\end{aligned}
$$

where $u_{0}$ is a given nonnegative constant, $\Lambda$ is a closed subset of a bounded domain $\Omega$ in $\mathbb{R}^{2}$ and $\partial_{n} u$ is the normal (to $\partial \Lambda$ ) outgoing derivative of $u$. This problem arises for instance from an obstacle problem (see for instance [24]).

THEOREM 1. - Assume that $a(0), f(0)>0$ and that $q \mapsto a(q), u \mapsto f(u)$ are increasing functions of class $C^{1}$ and $C^{0}$ respectively. If $\Omega \subset \mathbb{R}^{2}$ is convex and if $u$ is a solution of (1.1)-(1.3), then $\Lambda$ is also convex.

This theorem has been proved in the special case where $a$ and $f$ are constants by Friedman and Phillips [13] in two dimensions, and then extended to any dimension by Kawohl [19]. Similar results were also proved (in any dimensions) for $a \equiv 1, f \equiv 0$ and $\partial_{n} u=$ const $=\lambda>0$ in place of $\partial_{n} u=0$ by Caffarelli and Spruck [7]. The exterior problem in $\Omega=\mathbb{R}^{2} \backslash \mathcal{O}$ with $\mathcal{O}$ convex and $a \equiv 1$ has been studied for $\lambda>0$ by Kawohl [22] and Hamilton [14], and for $\lambda=0$ by Kawohl [21]. Let us also mention two related results on convex rings $[7,8]$ and, for general questions on the convexity of the level sets, [20]. We can also quote a recent paper by Caffarelli and Salazar [6] for the equation $\Delta u+c u=0$ and results by Henrot and Shahgholian [15-17] (which rely on a lower bound on the gradient), but for which the extension to general quasilinear operators has not yet be done. Concerning estimates on the curvature and the use of the Fréchet formula, one may refer to [25] (in the case of the Laplace operator). The results of this article were announced in [10].

We will prove Theorem 1 in a much more general framework, except that we will deal only with analytic solutions for reasons that will be made clear later. We will assume

$$
\partial_{n} u=\lambda(K) \geqslant 0 \quad \text { on } \partial \Lambda,
$$

where $\lambda$ is a function of the curvature $K$ of $\partial \Lambda$. Here we denote by $n$ and $\tau$ the normal and tangent unit vectors to a level set, so that $(\tau, n)$ is a direct orthonormal basis in $\mathbb{R}^{2}$, and $n=\frac{\nabla u}{|\nabla u|}$, if $\nabla u \neq 0$. In this case, the curvature is defined by $K=\frac{D_{\tau \tau} u}{|\nabla u|}$. We shall consider the analytic solutions of the fully nonlinear elliptic equation

$$
\mathcal{F}\left(D_{n n} u, D_{\tau \tau} u, D_{n \tau} u,|\nabla u|, u\right)=0 \quad \text { in } \Omega \backslash \Lambda,
$$

where $\mathcal{F}$ is an analytic function. The vectors $n$ and $\tau$ are well defined if $\nabla u \neq 0$. For the equation to make sense in case of a patch of zero gradient, we therefore require the following conditions.

(A0) Compatibility condition: we assume the existence of a function $\overline{\mathcal{F}}$ such that

$$
\mathcal{F}(a, b, c, 0, u)=\overline{\mathcal{F}}\left(a+b, a b-c^{2}, u\right) \quad \forall a, b, c, u .
$$


(A1) Nonzero gradient condition:

$$
\forall t \in\left(0, u_{0}\right], \quad \exists x \in \Omega \backslash \Lambda, \quad u(x)=t, \quad \nabla u(x) \neq 0 .
$$

Let us define $\alpha=(\mathcal{F})_{D_{n n} u}^{\prime}, \beta=(\mathcal{F})_{D_{\tau \tau} u}^{\prime}, \gamma=(\mathcal{F})_{D_{n \tau} u}^{\prime}$,

$$
\bar{\alpha}(x)=\inf _{a, b \in \mathbb{R}} \alpha(a, b, 0,|\nabla u(x)|, u(x)) \quad \text { and } \quad \bar{\beta}(x)=\inf _{a, b \in \mathbb{R}} \beta(a, b, 0,|\nabla u(x)|, u(x)) .
$$

(A2) Ellipticity conditions:

$$
\inf _{X} \bar{\alpha}>0, \quad \inf _{X} \bar{\beta}>0, \quad \inf _{X}\left(4 \alpha \beta-\gamma^{2}\right) \geqslant 0,
$$

where $X$ is the set of the points which realize the maximum of the gradient on their level line:

$$
X=\left\{x \in \Omega \backslash \Lambda:|\nabla u(x)|=\max _{\substack{y \in \Omega \backslash \Lambda \\ u(y)=u(x)}}|\nabla u(y)|\right\} .
$$

(A3) Condition on the free boundary: we assume that $\partial \Lambda$ is analytic and that the map $K \mapsto \lambda(K)$ is analytic nonincreasing. Moreover if $\lambda(K) \equiv 0$ on $\partial \Lambda$, then we assume that $\mathcal{F}(0,0,0,0,0)<0$ and that the vector field $n=\frac{\nabla u}{|\nabla u|}$ and the curvature $K$ are continuous up to $\partial \Lambda$.

These assumptions cover the case of Eq. (1.1) but also of quasilinear elliptic equations like the mean curvature equation

$$
\operatorname{div}\left(\frac{\nabla u}{\sqrt{1+|\nabla u|^{2}}}\right)=f(u,|\nabla u|)
$$

as well as fully nonlinear equations like the Monge-Ampère equation

$$
\operatorname{det}\left(D^{2} u\right)=f(u,|\nabla u|)>0 .
$$

From now on, we assume that $u$ is an analytic solution of Eq. (1.5) on $\Omega \backslash \Lambda$ (with eventually $\Lambda=\emptyset$ ).

Notations. - We shall note $B_{r}(x)$ the ball of center $x$ and radius $r>0$. For simplicity, we will use the same notation for a curve and its image. $\{F=0\}$ is the set $\{x \in$ $\Omega \backslash \Lambda: F(x)=0\}, \partial_{\sigma} u=\sigma \cdot \nabla u$ the derivative of $u$ along the unit vector $\sigma$ and $D_{\sigma \sigma} u:=\left(\sigma, D^{2} u \sigma\right)$. Since the tangent and normal unit vectors $\tau$ and $v$ depend on $x$, $\partial_{\tau}\left(\partial_{\tau} u\right) \neq D_{\tau \tau} u$ in general, and one has to use the Fréchet formula

$$
\begin{aligned}
\partial_{\tau} n & =K \tau, & \partial_{\tau} \tau & =-K n, \\
\partial_{n} n & =\rho \tau, & \partial_{n} \tau & =-\rho n,
\end{aligned}
$$

where $K=\frac{1}{|\nabla u|} D_{\tau \tau} u$ is the curvature of the level line and $\rho=\frac{1}{|\nabla u|} D_{n \tau} u$.

For $t>0$, let 


$$
\begin{gathered}
\Gamma^{t}=\{x \in \Omega: u(x)=t\}, \quad m(t)=\max _{y \in \Gamma^{t}}|\nabla u(y)|, \\
\text { and } \quad X^{t}=\left\{x \in \Gamma^{t}:|\nabla u(x)|=m(t)\right\} .
\end{gathered}
$$

With a straightforward abuse of notations, we define

$$
K(t):=\inf _{y \in X^{t}} \frac{D_{\tau \tau} u}{|\nabla u|}(y) .
$$

The following result is the core of our method.

THEOREM 2. - Under Assumptions (A0)-(A1)-(A2), consider an analytic solution $u$ of Eq. (1.5). With the above notations, $m$ is continuous and differentiable outside a countable closed set in $\left(0, u_{0}\right)$ such that

$$
\begin{gathered}
\mathcal{F}\left(m \frac{d m}{d t}, m K, 0, m, t\right)=0 \quad \text { for } t \in\left(0, u_{0}\right) \text { a.e. }, \\
\frac{d K}{d t} \leqslant-\frac{K^{2}}{m},
\end{gathered}
$$

where the inequality has to be understood in the sense of distributions.

Remark 1. - The method used in the proof of Theorem 2 has the following features.

(i) In higher dimensions, we can formally get a similar system for the mean curvature of the level sets and the maximal value of the gradient (see Appendix A.2).

(ii) In the case of a radially symmetric solution (when $\Omega$ is a ball), inequality (1.9) becomes an equality (see Appendix A.1). The result of Theorem 2 can therefore be compared with results based on rearrangement techniques, like the ones obtained by G. Talenti in another context [30].

(iii) In the nonradial case, we prove a refined version of (1.9):

$$
\frac{d K}{d t} \leqslant-\frac{K^{2}}{m}-\frac{1}{m}\left(2 \sqrt{\frac{\beta}{\alpha}}-\frac{|\gamma|}{\alpha}\right) \cdot \min _{X^{t}}\left|\partial_{\tau}\left(\frac{D_{\tau \tau} u}{|\nabla u|}\right)\right| .
$$

As a consequence of Theorem 2, we will prove our main result.

THEOREM 3. - Under Assumptions (A0)-(A3), if $u$ is an analytic (up to the fixed boundary $\partial \Omega$ ) solution to the free boundary problem (1.2)-(1.4), (1.5) (including the case $\Lambda=\emptyset)$, then $u$ has the following properties:

(i) There exists a constant $M$ which only depends on $\mathcal{F},|\lambda(K)|_{L^{\infty}(\partial \Lambda)}, u_{0}$ and the minimum of the signed curvature of $\partial \Omega$ such that $\|\nabla u\|_{L^{\infty}(\Omega \backslash \Lambda)} \leqslant M$.

(ii) The minimum of the signed curvature of $\partial \Lambda$ is bigger than the minimum of the signed curvature of $\partial \Omega$ :

$$
\inf _{\partial \Lambda} K \geqslant \inf _{\partial \Omega} K .
$$

As a consequence, if $\Omega$ is convex, each connected component of $\Lambda$ is also convex. 
Remark 2. - In Theorem 3, we get a global bound from below on the curvature of the free boundary. Note that in [29] D.G. Schaeffer proves (using a quasiconformal mapping) that for an obstacle problem of the type $\Delta u=f$, there exists a local bound from below on the curvature of the free boundary.

Also notice that the $L^{\infty}(\Omega \backslash \Lambda)$ bound is true for dimensions higher than 2 (see Remark 1 and Appendix A.2).

The rest of this paper is organized as follows. We will show in Section 2 that Theorem 1 is a consequence of Theorem 3. In Section 3, we prove Theorem 2 in a special case corresponding to the central idea of our approach, and then in full generality using a detailed analysis of the analytic structure of appropriate sets. With additional estimates near the free boundary, we obtain Theorem 3 in Section 4. The appendix is devoted to the much simpler setting of the radial case, the formal extension of our estimates to dimensions higher than 2 and technical results on analytic sets.

\section{Proof of Theorem 1}

We will start with perturbations of the level of the free boundary and considerations on its regularity. The method essentially goes as in [2], so we shall simply give a sketch of the proofs. Then we will prove that Theorem 1 is a consequence of Theorem 3. Before, simply notice that Eqs. (1.1)-(1.3) are such that Assumptions (A0)-(A3) are satisfied.

\subsection{A perturbation of the original problem (1.1)-(1.3)}

Consider

$$
\left\{\begin{array}{l}
\operatorname{div}\left(a\left(|\nabla u|^{2}\right) \nabla u\right)=f(u) \quad \text { in } \Omega \backslash \Lambda, \\
t=\left.u\right|_{\partial \Lambda}<u<\left.u\right|_{\partial \Omega}=u_{0}, \\
\partial_{n} u=0 \text { on } \partial \Lambda,
\end{array}\right.
$$

where $a$ and $f$ satisfy the assumptions of Theorem 1, and assume moreover that $\partial \Omega$ and $a, f$ are analytic.

We shall say that (2.1) has analytic solutions if $\partial \Lambda$ is analytic (see for instance [5]). Throughout this section, to emphasize the role of the level, $t$, we will denote by $u^{t}$ the corresponding solution and use the notation $\Lambda^{t}$ instead of $\Lambda$.

Let us start with a perturbation result.

PROPOSITION 1. - If $u^{t_{0}}$ is an analytic solution to the free boundary problem (2.1) for $t=t_{0}<u_{0}$, then there exists an $\eta>0$ such that (2.1) has analytic solutions for every $t \in\left(t_{0}-\eta, t_{0}+\eta\right)$. Moreover the map $t \mapsto \partial \Lambda^{t}$ is continuous (and $\partial \Lambda^{t}$ is analytic).

Sketch of the proof of Proposition 1. - From the assumption of Proposition 1, it follows that the boundaries $\partial \Omega$ and $\partial \Lambda^{t}$ are of class $C^{\infty}$. We can then apply Nash-Moser's inverse function Theorem as in [2] to prove that (2.1) has a solution $u^{t}$ for $t$ in a neighbourhood of $t_{0}$ with a smooth free boundary $\partial \Lambda^{t} \in C^{\infty}$. We conclude with the help of the following result on the regularity of the free boundary, due to Kinderlehrer and Nirenberg [23]: 
LEMMA 1. - Under the previous assumptions on the analytic problem (2.1), if the free boundary $\partial \Lambda^{t}$ is $C^{1}$ and $u^{t}$ is $C^{2}$ up to the free boundary, then the free boundary $\partial \Lambda^{t}$ is analytic.

Actually the perturbation result also holds in a neighborhood of $u_{0}$.

PROPOSITION 2. - There exists an $\eta>0$, such that for every $t$ in $\left(u_{0}-\eta, u_{0}\right]$, the free boundary problem (2.1) has an analytic solution. Moreover the map $t \mapsto \partial \Lambda^{t}$ is continuous (and $\partial \Lambda^{t}$ is analytic).

Sketch of the proof of Proposition 2. - For $t=u_{0}$, the function $u^{t} \equiv u_{0}$ is a solution with $\Lambda^{t}=\bar{\Omega}$. This problem is then degenerate in $t=u_{0}$. Nevertheless, as in [2], we can apply a Nash-Moser approach in this degenerate case, which proves Proposition 2.

\subsection{Proof of Theorem 1}

The main advantage of the obstacle problem (1.1)-(1.3) compared to the more general free boundary problem (1.4) is that it is known that there exists a unique weak solution (see $[28,12]$ ), and that this solution is bounded in $W^{2, \infty}$ (see $[12,11,3,1]$ ). As a consequence of the uniqueness, the map $t \mapsto u^{t} \in W^{2, p}$ is continuous for every $p \in(1,+\infty)$. Moreover, from the nondegeneracy lemma (see Caffarelli [4], and for instance [26]), we have the

LEMMA 2. - Consider a solution of problem (1.1)-(1.3). Under the assumptions of Theorem 1 , for every $t_{0} \in\left[0, u_{0}\right]$,

$$
\begin{gathered}
\lim _{\substack{t \rightarrow t_{0} \\
t \in\left[0, u_{0}\right]}} \Lambda^{t}=\Lambda^{t_{0}} \\
\text { and }\left|\partial \Lambda^{t_{0}}\right|=0 .
\end{gathered}
$$

Let us prove that $t^{*}$ defined by

$$
t^{*}=\inf \left\{t_{0} \in\left(0, u_{0}\right): \forall t \in\left[t_{0}, u_{0}\right),(1.1)-(1.3) \text { has an analytic solution } u^{t}\right\}
$$

is actually 0 . Because of Proposition $1, t^{*}$ is the infimum of a nonempty set. Assume by contradiction that $t^{*}>0$. From Theorem 3, we deduce that $\inf _{\partial \Lambda^{t}} K \geqslant \inf _{\partial \Omega} K \geqslant 0$ for $t \in\left(t^{*}, u_{0}\right)$ and then $\Lambda^{t}$ is convex: by continuity (Lemma $\left.2,(2.2)\right), \Lambda^{t^{*}}$ is also convex.

(1) Case $\operatorname{Int}\left(\Lambda^{t^{*}}\right)=\emptyset:\left|\Lambda^{t^{*}}\right|=\left|\partial \Lambda^{t^{*}}\right|=0$ from Lemma 2, (2.3). In this case there is no free boundary, i.e. the solution $u^{t}$ satisfies the Euler-Lagrange equation (1.1) of the energy

$$
\mathcal{E}(u)=\int_{\Omega}\left(\frac{1}{2} A\left(|\nabla u|^{2}\right)+G(u)\right) d x
$$

without constraints, where $A^{\prime}=a, G^{\prime}=f$. The uniqueness of the weak solution to the free boundary problem consequently implies that $u^{t^{*}}=u$, and because we assumed that $\min _{\Omega} u=0$, we get $t^{*}=0$, a contradiction.

(2) Case $\operatorname{Int}\left(\Lambda^{t^{*}}\right) \neq \emptyset$ : we use the following result, due to Caffarelli [4]. 
LEMMA 3. - Under the previous assumptions on the obstacle problem (1.1)-(1.3), if the coincidence set $\Lambda^{t}$ is convex and if $\operatorname{Int}\left(\Lambda^{t}\right) \neq \emptyset$, then $\partial \Lambda^{t}$ is $C^{1}$ and $u^{t}$ is $C^{2}$ up to the free boundary $\partial \Lambda^{t}$.

Lemma 1 therefore implies that the free boundary is analytic. Finally $u^{t^{*}}$ is an analytic solution, and Proposition 1 gives a contradition with the definition of $t^{*}$.

This proves Theorem 1 in the context of analytic solutions. Now because the solution is the limit of an approximating sequence of analytic solutions of a regularized problem, the result holds as well if $a$ and $f$ are only of class $C^{1}$ and $C^{0}$ respectively. This ends the proof of Theorem 1 .

Remark 4. - This last argument of approximation applies when existence and uniqueness results can be proved, which is true for the obstacle problem of Theorem 1 but is not known for more general problems (1.2)-(1.4), (1.5).

Also notice that the convexity of the free boundary holds for any solution which can be seen as the limit of analytic solutions of aproximating problems. This is a method to get an existence result of solutions with convex free boundaries (see for instance [25] in the case of the Laplace operator).

\section{Proof of Theorem 2}

First we shall assume that locally in $t, X^{t}$ (which is the set of the points of the level line $\Gamma^{t}$ of $u$ which realize the maximum of $\left.|\nabla u|\right)$ is supported in an analytic curve $t \mapsto x^{t}$ such that $t=u\left(x^{t}\right)$. The justification of such an assumption will be given in the next subsection.

\subsection{Proof of Theorem 2 in a particular case}

Let $x^{t}$ be a point where the maximum of the gradient is reached on a level line $\Gamma^{t}$ :

$$
\left\{\begin{array}{l}
\bar{F}(t):=\left.\partial_{\tau}\left(\frac{1}{2}|\nabla u|^{2}\right)\right|_{x=x^{t}}=0, \\
\bar{G}(t):=\left.\partial_{\tau}^{2}\left(\frac{1}{2}|\nabla u|^{2}\right)\right|_{x=x^{t}} \leqslant 0 .
\end{array}\right.
$$

According to the definition of the normal and tangent unit vectors $n$ and $\tau$, we get, at $x=x^{t}$,

$$
\bar{F}(t)=|\nabla u| D_{n \tau} u \quad \text { and } \quad \bar{G}(t)=\left(\partial_{\tau}|\nabla u|\right) D_{n \tau} u+|\nabla u| \partial_{\tau}\left(D_{n \tau} u\right) .
$$

With the definition $m(t):=\left|\nabla u\left(x^{t}\right)\right|>0$ by (A1), this can be rewritten as

$$
\left\{\begin{array}{l}
\overline{\bar{F}}(t)=D_{n \tau} u\left(x^{t}\right)=0, \\
\bar{G}(t)=\left.m \partial_{\tau}\left(D_{n \tau} u\right)\right|_{x=x^{t}} \leqslant 0 .
\end{array}\right.
$$

Equation for $m$ : deriving the identity $u\left(x^{t}\right)=t$ with respect to $t$, which can certainly be done at least if $t \mapsto x^{t}$ is an analytic curve, we get

$$
n \cdot \frac{d x^{t}}{d t}=\frac{1}{m}
$$


so that $m \frac{d m}{d t}=\frac{d}{d t}\left(\frac{1}{2}\left|\nabla u\left(x^{t}\right)\right|^{2}\right)=\nabla u\left(x^{t}\right) \cdot D^{2} u \cdot \frac{d x^{t}}{d t}=D_{n n} u\left(x^{t}\right)$. Using the curvature $K=\frac{D_{\tau \tau} u}{|\nabla u|}$, we may write $D_{\tau \tau} u=m K$, and Eq. (1.5) at $x=x^{t}$ gives Eq. (1.8).

Inequation for $K$ : we compute $\frac{d K}{d t}=\frac{1}{m} \partial_{n} K+h \partial_{\tau} K$, where $h=\tau \cdot \frac{d x^{t}}{d t}$. To get an expression of $h$, we derive $\frac{\bar{F}}{m}$ with respect to $t$ :

$$
0=\frac{d}{d t}\left(\frac{\bar{F}}{m}(t)\right)=\frac{1}{m} \partial_{n}\left(D_{n \tau} u\right)+h \partial_{\tau}\left(D_{n \tau} u\right) .
$$

Because of the Fréchet formula (1.6) and (1.7), and using the fact that $D_{n \tau} u\left(x^{t}\right)=0$, we have

$$
\left\{\begin{array}{l}
\partial_{\tau} K:=\partial_{\tau}\left(\frac{D_{\tau \tau} u}{|\nabla u|}\right)=\frac{1}{|\nabla u|} D_{\tau \tau \tau} u, \\
\partial_{n} K:=\partial_{n}\left(\frac{D_{\tau \tau} u}{|\nabla u|}\right)=\frac{1}{|\nabla u|} \partial_{\tau}\left(D_{n \tau} u\right)-\left(\frac{D_{\tau \tau} u}{|\nabla u|}\right)^{2}, \\
\partial_{n}\left(D_{n \tau} u\right)=D_{n n \tau} u .
\end{array}\right.
$$

To evaluate $D_{n n \tau} u$, we derive Eq. (1.5) with respect to $\tau$ :

$$
\alpha D_{n n \tau} u+\beta D_{\tau \tau \tau} u+\gamma \partial_{\tau}\left(D_{n \tau} u\right)=0 .
$$

If $\bar{G} \neq 0$, putting these expressions all together, we get

$$
\frac{d K}{d t}=-\frac{K^{2}}{m}+\frac{\gamma}{\alpha}\left(\frac{\partial_{\tau} K}{m}\right)+\frac{\beta}{\alpha} m\left(\partial_{\tau} K\right)^{2} \frac{1}{\bar{G}}+\frac{\bar{G}}{m^{3}},
$$

and an optimization on $\bar{G}<0$ gives

$$
\frac{d K}{d t} \leqslant-\frac{K^{2}}{m}-\frac{1}{m}\left(2 \sqrt{\frac{\beta}{\alpha}}-\frac{|\gamma|}{\alpha}\right)\left|\partial_{\tau} K\right| .
$$

If $\bar{G}=0$, then it is easy to see that $\partial_{\tau} K=0$ and $\frac{d K}{d t}=-\frac{K^{2}}{m}$. In any case, (3.1) is true, which proves inequality (1.9).

\subsection{Proof of Theorem 2 in the general case}

We will introduce analytic functions relevant to our problem and then give the proof of Theorem 2 in this framework. Here is the technical part of the proof, for which we shall distinguish two cases.

Let us recall that a point $x^{t}$ belongs to $X^{t}$ if and only if $u\left(x^{t}\right)=t$ and $\left|\nabla u\left(x^{t}\right)\right|=$ $\max _{\{y \in \Omega: u(y)=t\}}|\nabla u(y)|$. This implies that

$$
\left.\frac{d}{d \tau}\left(|\nabla u|^{2} / 2\right)\right|_{x=x^{t}}=0
$$

Now let us define on $\{|\nabla u|>0\}$ the analytic function:

$$
F(x)=\left.\frac{d}{d \tau}\left(|\nabla u|^{2} / 2\right)\right|_{x}
$$


(with the notations of Section 3.1, $F(t)=F\left(x^{t}\right)=0$ ). Let $X=\bigcup_{t \in\left[0, u_{0}\right]} X^{t}$. From (3.2), we know that $X \subset\{F=0\}$ is an analytic set, if we define analytic sets as sets where analytic functions vanish.

Case A: $F \equiv 0$ : this is the simplest case (see Appendix A.1 for details).

LEMMA 4. - If $F \equiv 0$, then $\Omega$ is a disk and $u$ is radially symmetric.

Proof. - If $F \equiv 0$, then $|\nabla u|=$ const $=m(t)$ on each level line $\Gamma^{t}=\{u=t\}$. Let $\gamma$ be a smooth curve such that $u(\gamma(t))=t$. Because here $X=\Omega \backslash \Lambda$, any such curve can be seen as a curve $t \mapsto x^{t}$ used in the previous subsection. As a consequence we have

$$
\mathcal{F}\left(m \frac{d m}{d t}, m K, 0, m, t\right)=0
$$

where $K=K(\gamma(t))$. From Assumption (A2), we deduce that $K=K(t)$ on $\Gamma^{t}$. Because $\Omega$ is bounded, the level lines of $u$ are circles.

Moreover $\partial_{n} n=m^{-1} D_{n \tau} u \tau=0$. This implies that if $x_{1} \in \partial \Omega$ and $\gamma_{x_{1}}$ is the integral curve of the vector field $n$ such that $u\left(\gamma_{x_{1}}(t)\right)=t$ and $\gamma_{x_{1}}\left(u_{0}\right)=x_{1}$, then $\frac{d}{d t} n\left(\gamma_{x_{1}}(t)\right)=0$ :

$$
\gamma_{x_{1}}(t)=x_{1}+\left(t-u_{0}\right) n\left(x_{1}\right) \quad \text { and } \quad u\left(\gamma_{x_{1}}(t)\right)=t
$$

Because this is true for every point $x_{1}$ in the circle $\partial \Omega$, we see that the circles $\Gamma^{t}$ have the same center $x_{0}$. In particular the solution is radial on the annulus $\Omega \backslash \Lambda=$ $B_{1 / K\left(u_{0}\right)}\left(x_{0}\right) \backslash B_{1 / K(0)}\left(x_{0}\right)$.

Case B: $F \not \equiv 0$ : we begin with a statement that will be proved in Appendix A.3.

LEMMA 5. - For every $\varepsilon>0$, for $m(t)=\sup _{\{u=t\}}|\nabla u|$, let $\omega_{\varepsilon}$ be defined by

$$
\omega_{\varepsilon}=\left\{x \in \Omega: \varepsilon<u(x)<u_{0}-\varepsilon,|\nabla u(x)|>\varepsilon m(u(x))\right\} .
$$

Then there exists an open set $\omega$ which is a finite union of balls such that

$$
\{F=0\} \cap \omega_{\varepsilon} \subset \omega:=\bigcup_{i=1}^{N} B_{r_{i}}\left(x_{i}\right) \subset\{|\nabla u|>0\} .
$$

Moreover the set $F_{\omega}:=\{F=0\} \cap \omega$ has the following property:

$$
\forall i \in[1, N], \quad \exists k_{i} \in \mathbb{N}, \quad F_{\omega} \cap B_{r_{i}}\left(x_{i}\right)=\left\{x_{i}\right\} \cup\left(\bigcup_{j=1}^{k_{i}} \gamma_{j}^{i}\right),
$$

where $\gamma_{j}^{i}$ are analytic open curves with $x_{i}$ as origin such that in a neighborhood of a singular point $x_{i}$, either $\frac{d}{d s}\left(u \circ \gamma_{j}^{i}\right)=0$ or (up to change the parametrization) $\frac{d}{d s}\left(u \circ \gamma_{j}^{i}\right)>0$. Here $s$ is the curvilinear coordinate.

As a consequence of the lemma we get that for any $\varepsilon>0$ small enough, $X \cap\{\varepsilon<u<$ $\left.u_{0}-\varepsilon\right\}$ is contained in $F_{\omega}$ which has an analytic structure given by (3.3). 
We are now going to prove that (1.8) and (1.9), which have been established in the case of an analytic curve, are also valid in the general framework.

Let us assume that $\varepsilon>0$ is fixed in all what follows. Denote by $\gamma$ the generic curve defined in Lemma 5 and let $\left\{x_{i}^{\prime}\right\}$ be the set of points on the curves $\gamma$ such that $\frac{d}{d s}(u \circ \gamma) \not \equiv 0$ and $\left.\frac{d}{d s}(u \circ \gamma)\right|_{\gamma(s)=x_{i}^{\prime}}=0$. Because the curves $\gamma$ and the function $u$ are analytic, we deduce that there are only a finite number $N^{\prime}$ of such points $x_{i}^{\prime}$. Let $\mathcal{G}$ and $\mathcal{G}_{0}$ be the sets of curves $\gamma$ such that $\frac{d}{d s}(u \circ \gamma)>0$ and $u(\gamma)=$ const respectively. Then

$$
\{F=0\} \cap \omega=\mathcal{G} \cup \mathcal{G}_{0} \cup\left\{x_{i}\right\}_{1 \leqslant i \leqslant N} \cup\left\{x_{i}^{\prime}\right\}_{1 \leqslant i \leqslant N^{\prime}} .
$$

We can rewrite the discret set $\left\{u\left(x_{i}\right)\right\}_{1 \leqslant i \leqslant N} \cup\left\{u\left(x_{i}^{\prime}\right)\right\}_{1 \leqslant i \leqslant N^{\prime}} \cup\{u(\gamma)\}_{\gamma \in \mathcal{G}_{0}}$ as an increasing finite sequence of critical values, $t_{k}^{*} \in\left(\varepsilon, u_{0}-\varepsilon\right), k=1,2, \ldots, M$. Let $\mathcal{G}_{k}$ be the set of curves $\gamma \in \mathcal{G}$ which range in $\left\{t_{k}^{*}<u<t_{k+1}^{*}\right\}$. Then on $\left(t_{k}^{*}, t_{k+1}^{*}\right)$ we have:

$$
m(t)=\sup _{\gamma \in \mathcal{G}_{k}}|\nabla u(\gamma(t))| .
$$

Because each map $t \mapsto|\nabla u(\gamma(t))|$ is analytic, we deduce from Proposition 4 that this supremum is analytic except maybe on a discret set $\left\{t_{k, n}^{*}\right\}_{p_{k}^{-}<n<p_{k}^{+}}$with $p_{k}^{-}, p_{k}^{+} \in$ $\mathbb{Z} \cup\{-\infty\} \cup\{+\infty\}$, which has no accumulation point in $\left(t_{k}^{*}, t_{k+1}^{*}\right)$. Only $t_{k}^{*}$ and $t_{k+1}^{*}$ are possible accumulation points. In particular there exists $\gamma_{t_{k, n}} \in \mathcal{G}_{k}$ such that

$$
m(t)=\left|\nabla u\left(\gamma_{t_{k, n}}(t)\right)\right| \quad \text { on }\left(t_{k, n}, t_{k, n+1}\right) .
$$

Then the proof of Theorem 2, given above in the special case where $X^{t}$ is supported in an analytic curve $t \mapsto x^{t}=\gamma_{t_{k, n}}(t)$, applies and gives the equations written in Theorem 2 for

$$
K(t)=K\left(\gamma_{t_{k, n}}(t)\right) \quad \text { on }\left(t_{k, n}, t_{k, n+1}\right) .
$$

Although the map $t \mapsto m(t)$ is continuous, the map $t \mapsto K(t)$ can be discontinuous in $t_{k, n}$. In other words we can have $K\left(\gamma_{t_{k, n-1}}\left(t_{k, n}\right)\right) \neq K\left(\gamma_{t_{k, n}}\left(t_{k, n}\right)\right)$. Nevertheless we have the

LEMMA 6. - With the above notations,

$$
K\left(\gamma_{t_{k, n}}\left(t_{k, n}\right)\right) \leqslant K\left(\gamma_{t_{k, n-1}}\left(t_{k, n}\right)\right) .
$$

We can therefore define $K\left(t_{k, n}\right):=K\left(\gamma_{t_{k, n}}\left(t_{k, n}\right)\right)$ and then (1.10) is true in the sense of distribution on $\left(t_{k, n-1}, t_{k, n+1}\right)$ :

$$
\dot{K} \leqslant-\frac{K^{2}}{m}-\frac{1}{m}\left(2 \sqrt{\frac{\alpha}{\beta}}-\frac{|\gamma|}{\beta}\right)\left|\partial_{\tau} K\right|,
$$

where $\partial_{\tau} K$ is taken on $\gamma_{t_{k, n-1}}$ for $t<t_{k, n}$ and $\gamma_{t_{k, n}}$ for $t>t_{k, n}$.

Proof of Lemma 6. - Let us recall that for each curve $\gamma=\gamma_{t_{k, n-1}}$ and $\gamma=\gamma_{t_{k, n}}$ we have

$$
\mathcal{F}\left(m \frac{d m}{d t}, m K, 0, m, t\right)=0
$$


where $m(t)=|\nabla u(\gamma(t))|$ and $K(t)=K(\gamma(t))$. Let $m^{+}(t)=\left|\nabla u\left(\gamma_{t_{k, n}}(t)\right)\right|$ and $m^{-}(t)=$ $\left|\nabla u\left(\gamma_{t_{k, n-1}}(t)\right)\right|$. Then

$$
m^{ \pm}(t)=m\left(t_{k, n}\right)+l^{ \pm} \cdot\left(t-t_{k, n}\right)+\mathrm{o}\left(\left|t-t_{k, n}\right|\right)
$$

where $l^{ \pm}=\left.\frac{d m^{ \pm}(t)}{d t}\right|_{t=t_{k, n}}$. Note that because of Assumption (A2), $l^{+}-l^{-}$has the same sign as $-K\left(\gamma_{t_{k, n}}\left(t_{k, n}\right)\right)+K\left(\gamma_{t_{k, n-1}}\left(t_{k, n}\right)\right)$. Because of

$$
m(t)=\sup \left(m^{+}(t), m^{-}(t)\right)= \begin{cases}m^{+}(t) & \text { on }\left(t_{k, n}, t_{k, n+1}\right) \\ m^{-}(t) & \text { on }\left(t_{k, n-1}, t_{k, n}\right)\end{cases}
$$

we deduce that $l^{+} \geqslant l^{-}$and then $K\left(\gamma_{t_{k, n}}\left(t_{k, n}\right)\right) \leqslant K\left(\gamma_{t_{k, n-1}}\left(t_{k, n}\right)\right)$ which ends the proof of Lemma 6.

More generally (3.5) and (3.4) are true on $\left(t_{k}^{*}, t_{k+1}^{*}\right)$ for $K$ defined by

$$
K(t)=\inf _{x^{t} \in X^{t}} K\left(x^{t}\right)
$$

We now want to prove that these equations are still true in a neighborhood of a critical value $t_{k}^{*}$. It is clear that (3.5) is true almost everywhere in $\left(t_{k-1}^{*}, t_{k+1}^{*}\right)$, because the map $t \mapsto m(t)$ is continuous. We have to prove that (3.4) is true on $\left(t_{k-1}^{*}, t_{k+1}^{*}\right)$ in the sense of distributions. Let $\phi \in C_{0}^{\infty}\left(t_{k-1}^{*}, t_{k+1}^{*}\right), \phi \geqslant 0$ :

$$
\begin{aligned}
\langle\dot{K}, \phi\rangle= & -\int_{t_{k-1}^{*}}^{t_{k+1}^{*}} K \dot{\phi} \\
= & -\lim _{\delta \rightarrow 0}\left\{\int_{t_{k-1}^{*}}^{t_{k}^{*}-\delta} K \dot{\phi}+\int_{t_{k}^{*++\delta}}^{t_{k+1}^{*}} K \dot{\phi}\right\} \\
= & -\lim _{\delta \rightarrow 0}\left\{\int_{t_{k-1}^{*}}^{t_{k}^{*}-\delta}-\dot{K} \phi+\int_{t_{k}^{*}+\delta}^{t_{k+1}^{*}}-\dot{K} \phi+[K \phi]_{t_{k-1}^{*}-\delta}^{t_{k}^{*}-\delta}+[K \phi]_{t_{k}^{*}+\delta}^{t_{k+1}^{*}}\right\} \\
\leqslant & \int_{t_{k-1}^{*}}^{t_{k+1}^{*}}\left(-\frac{K^{2}}{m}-\frac{1}{m}\left(2 \sqrt{\frac{\alpha}{\beta}}-\frac{|\gamma|}{\beta}\right)\left|\partial_{\tau} K\right|\right) \phi \\
& +\phi\left(t_{k}^{*}\right) \lim _{\delta \rightarrow 0} \sup \left(K\left(t_{k}^{*}+\delta\right)-K\left(t_{k}^{*}-\delta\right)\right) \\
\leqslant & \left\langle-\frac{K^{2}}{m}-\frac{1}{m}\left(2 \sqrt{\frac{\alpha}{\beta}}-\frac{|\gamma|}{\beta}\right)\left|\partial_{\tau} K\right|, \phi\right\rangle
\end{aligned}
$$

because $\phi\left(t_{k}^{*}\right) \geqslant 0$ and because of the 
LEMMA 7. - With the above notations,

$$
\limsup _{\delta \rightarrow 0}\left(K\left(t_{k}^{*}+\delta\right)-K\left(t_{k}^{*}-\delta\right)\right) \leqslant 0
$$

Lemma 7 is a kind of generalization of Lemma 6 that will be proved in Appendix A.3. The system (3.4)-(3.5) holds on $\left(t_{k-1}^{*}, t_{k+1}^{*}\right)$ and then on $(t+\varepsilon, \bar{t}-\varepsilon)$. Taking the limit $\varepsilon \rightarrow 0$, we end up with the proof of Theorem 2 .

\section{Proof of Theorem 3}

The proof of Theorem 3 relies mainly in the fact that as $t \rightarrow 0$, the set $X^{t}$ accumulates in a point of $\partial \Lambda$ which realizes the minimum of the curvature of the free boundary.

PROPOSITION 3. - Under the assumptions of Theorem 3, let $x^{t}$ be a point such that

$$
u\left(x^{t}\right)=t \quad \text { and } \quad\left|\nabla u\left(x^{t}\right)\right|=\max _{u(y)=t}|\nabla u(y)|
$$

If $x^{t} \rightarrow x^{0}$ as $t \rightarrow 0$, then $K\left(x^{t}\right) \longrightarrow K\left(x^{0}\right)=\inf _{x \in \partial \Lambda} K(x)$.

\subsection{End of the proof of Theorem 3}

Property (ii) of Theorem 3 follows from Theorem 2, (1.9) and Proposition 3. To prove (i), we first remark that because of Assumption (A2), we can rewrite (1.8) as

$$
\frac{d\left(m^{2}\right)}{d t}=\mathcal{H}(m, K, t) \quad \text { on }\left(0, u_{0}\right),
$$

where $\mathcal{H}$ is analytic with respect to $(m, K, t) \in[0,+\infty) \times \mathbb{R} \times\left[0, u_{0}\right]$ and decreasing with respect to $K$. Because of the inequality on $K$, it is clear that $K(t) \geqslant \inf _{\partial \Omega} K=: K_{0}$. Thus we get

$$
\left\{\begin{array}{l}
\frac{d\left(m^{2}\right)}{d t} \leqslant \mathcal{H}\left(m, K_{0}, t\right) \quad \text { a.e. on }\left(0, u_{0}\right), \\
m(0)=|\lambda(K)|_{L^{\infty}(\partial \Lambda)} .
\end{array}\right.
$$

We can then compare with the solution $m_{0}$ of

$$
\left\{\begin{array}{l}
\frac{d\left(m_{0}^{2}\right)}{d t}=\mathcal{H}\left(m_{0}, K_{0}, t\right) \quad \text { on }\left(0, u_{0}\right) \\
m(0)=|\lambda(K)|_{L^{\infty}(\partial \Lambda)}+1 .
\end{array}\right.
$$

The uniqueness of $m_{0}$ and its local existence are clear. Using the fact that $m>0$ on $\left(0, u_{0}\right)$, it is quite classical to see that if $m_{0} \geqslant m$ on $(a, b) \subset \subset\left(0, u_{0}\right)$, then $m_{0}$ is defined on $\left(a, u_{0}\right)$ and satisfies $m_{0} \geqslant m$ on $\left(a, u_{0}\right)$. On the other hand by the continuity of $m$ in $t=0$, we get that $m_{0}>m$ on a small interval $[0, \varepsilon)$, which implies that $m_{0} \geqslant m$ on $\left[0, u_{0}\right]$. In conclusion, the solution $m_{0}$ exists on $\left[0, u_{0}\right]$, is unique and is an upper bound for $m$ on $\left[0, u_{0}\right]$. This ends the proof of Theorem 3 . 


\subsection{Proof of Proposition 3}

First let us recall that according to Morrey [27], the solution $u$ is analytic up to the free boundary $\partial \Lambda$, because $\partial \Lambda$ is analytic itself. We will now distinguish three cases.

Case $\lambda\left(\inf _{\partial \Omega} K\right)>0$ and $\lambda(K) \not \equiv$ constant on $\partial \Lambda$ : according to Assumption (A3) $\lambda$ is assumed to be analytic nondecreasing in $K$. Thus $\max _{\partial \Lambda} \lambda(K)=\lambda\left(\inf _{\partial \Lambda} K\right)>0$ and by continuity, Proposition 3 is true.

Case $\lambda=$ constant $>0$ on $\partial \Lambda$ : let $x_{0} \in \partial \Lambda$ and $\gamma_{x_{0}}(t)$ be the integral curve of the vector field $n$ such that $\gamma_{x_{0}}(0)=x_{0}$ and $u\left(\gamma_{x_{0}}(t)\right)=t$ :

$$
\gamma_{x_{0}}(t)=x_{0}+\frac{t}{\lambda} n\left(x_{0}\right)+\mathrm{O}\left(t^{2}\right) \quad \text { for } t \geqslant 0 .
$$

For $t>0$ close enough to 0 , the map $x_{0} \mapsto \gamma_{x_{0}}(t)$ is a diffeomorphism from $\partial \Lambda$ onto $\Gamma^{t}=\{u=t\}$. Then for every $x \in \Gamma^{t}$ with $t>0$ close to 0 , there exists a unique $x_{0} \in \partial \Lambda$ such that $x=\gamma_{x_{0}}(t)$, and $\mid \nabla u\left(\gamma_{x_{0}}(t) \mid=\lambda+\frac{t}{\lambda} D_{n n} u\left(x_{0}\right)+\mathrm{O}\left(t^{2}\right)\right.$ can be inverted into

$$
D_{n n} u\left(x_{0}\right)=\frac{\lambda}{t}\left(\left|\nabla u\left(\gamma_{x_{0}}(t)\right)\right|-\lambda\right)+\mathrm{O}(t) .
$$

Moreover $D_{n \tau} u\left(\gamma_{x_{0}}(t)\right)=\mathrm{O}(t)$. Thus we get

$$
\mathcal{F}\left(\frac{\lambda}{t}\left(\left|\nabla u\left(\gamma_{x_{0}}(t)\right)\right|-\lambda\right), \lambda K\left(\gamma_{x_{0}}(t)\right), 0, \lambda, t\right)=\mathrm{O}(t) .
$$

Now for $t>0$ small enough and $x^{t} \in X^{t}$, let $x_{0}^{t} \in \partial \Lambda$ be defined by $x^{t}=\gamma_{x_{0}^{t}}(t)$ :

$$
\frac{1}{t}\left(\left|\nabla u\left(\gamma_{x_{0}^{t}}(t)\right)\right|-\lambda\right)=\frac{1}{t}\left(\sup _{x_{0} \in \partial \Lambda}\left|\nabla u\left(\gamma_{x_{0}}(t)\right)\right|-\lambda\right) .
$$

From Assumption (A2), we can deduce that

$$
\forall x_{0} \in \partial \Lambda, \quad K\left(\gamma_{x_{0}}(t)\right) \geqslant K\left(\gamma_{x_{0}^{t}}(t)\right)+\mathrm{O}(t) .
$$

Up to extraction of some subsequence, we can assume that $x_{0}^{t}$ converges to some $x_{0}^{0} \in \partial \Lambda$ as $t \rightarrow 0^{+}$. Because $\lambda>0$ on $\partial \Lambda$, the continuity of the curvature $K$ up to the free boundary $\partial \Lambda$ is automatically satisfied. We then deduce by continuity that $K\left(x_{0}^{0}\right)=\inf _{\partial \Lambda} K$, which proves Proposition 3.

Case $\lambda \equiv 0$ on $\partial \Lambda$ : we first remark that deriving $\partial_{n} u(x)=0$ on $\partial \Lambda$ with respect to the tangential to $\partial \Lambda$ vector field $\tau$, we get

$$
D_{n \tau} u=0 \quad \text { on } \partial \Lambda \text {. }
$$

As $x \rightarrow x_{0} \in \partial \Lambda$, by passing to the limit in (1.5), we get $\mathcal{F}\left(D_{n n} u\left(x_{0}\right), 0,0,0,0\right)=0$. Because $\mathcal{F}(0,0,0,0,0)<0, D_{n n} u\left(x_{0}\right)$ is the unique positive root of $s \mapsto \mathcal{F}(s, 0,0,0,0)$. The function $D_{n n} u$ is therefore constant on $\partial \Lambda$. 
For $x$ close to $\partial \Lambda$, we set $h=d(x, \partial \Lambda)$ and associate $x_{0}$ to $x$ as follows

$$
x=\gamma_{x_{0}}(h) \quad \text { and } \quad \gamma_{x_{0}}(h)=x_{0}+h n\left(x_{0}\right)
$$

For $h$ small enough, the map $\left(x_{0}, h\right) \mapsto \gamma_{x_{0}}(h)$ is indeed a local diffeomorphism. In place of $n$, consider the vector field $n_{0}(x)=n\left(x_{0}(x)\right)$.

$$
\begin{aligned}
u\left(\gamma_{x_{0}}(h)\right) & =\int_{0}^{h} D_{n_{0}} u\left(x_{0}+s n_{0}\right) d s \\
& =\int_{0}^{h} d s\left(D_{n_{0}} u\left(x_{0}\right)+s D_{n_{0} n_{0}} u\left(x_{0}\right)+\frac{s^{2}}{2} D_{n_{0} n_{0} n_{0}} u\left(x_{0}\right)+\mathrm{O}\left(s^{3}\right)\right)
\end{aligned}
$$

gives

$$
t=\frac{h^{2}}{2} D_{n_{0} n_{0}} u\left(x_{0}\right)+\frac{h^{3}}{6} D_{n_{0} n_{0} n_{0}} u\left(x_{0}\right)+\mathrm{O}\left(h^{4}\right) .
$$

We deduce that

$$
h=t^{1 / 2}\left(\frac{2}{D_{n_{0} n_{0}} u}\right)^{1 / 2}-\frac{t}{3} \frac{D_{n_{0} n_{0} n_{0}} u}{\left(D_{n_{0} n_{0}} u\right)^{2}}+\mathrm{O}\left(t^{3 / 2}\right)
$$

and a computation (repeated indices are summed) gives with $y=\gamma_{x_{0}}(h)-x_{0}$ :

$$
\begin{aligned}
\left|\nabla u\left(\gamma_{x_{0}}(h)\right)\right|^{2}= & \left|\nabla u\left(x_{0}\right)\right|^{2}+2 \nabla_{i} u D_{i j}^{2} u \cdot y_{j} \\
& +\frac{1}{2}\left\{D_{i j} u D_{i k} u+2 \nabla_{i} u D_{i j k} u\right\} y_{j} y_{k} \\
& +\frac{1}{6}\left\{6 D_{i j k} u D_{i l} u+2 \nabla_{i} u D_{i j k l} u\right\} y_{j} y_{k} y_{l}+\mathrm{O}\left(|y|^{4}\right) \\
= & \left(D_{n_{0} n_{0}} u\right)^{2} h^{2}+\left(D_{n_{0} n_{0}} u\right)\left(D_{n_{0} n_{0} n_{0}} u\right) h^{3}+\mathrm{O}\left(|y|^{4}\right) \\
= & 2 t\left(D_{n_{0} n_{0}} u\right)^{2}+\frac{4}{3} t^{3 / 2}\left(D_{n_{0} n_{0} n_{0}} u\right)\left(\frac{2}{D_{n_{0} n_{0}} u}\right)^{1 / 2}+\mathrm{O}\left(t^{2}\right) .
\end{aligned}
$$

LEMMA 8. - With the above notations,

$$
D_{n_{0} n_{0} n_{0}} u\left(x_{0}\right)=-D_{n_{0} n_{0}} u\left(x_{0}\right)\left(\frac{\beta}{\alpha} K\left(x_{0}\right)+\frac{\delta}{\alpha}\right) \quad \text { on } \partial \Lambda \text { where } \delta=\mathcal{F}^{\prime}|\nabla u|
$$

Combining the above computations, we get

$$
\left|\nabla u\left(\gamma_{x_{0}}(h)\right)\right|^{2}=2 t\left(D_{n_{0} n_{0}} u\right)^{2}-\frac{4}{3} t^{3 / 2}\left(\frac{\beta}{\alpha} K\left(x_{0}\right)+\frac{\delta}{\alpha}\right)\left(2 D_{n_{0} n_{0}} u\right)^{1 / 2}+\mathrm{O}\left(t^{2}\right) .
$$

We see that the gradient is maximum when the curvature is minimal, which ends the proof of Proposition 3. 
Proof of Lemma 8. - First, let us recall the following relations:

$$
\partial_{n_{0}} n=\left(\frac{D_{\tau n_{0}} u}{|\nabla u|}\right) \cdot \tau, \quad \partial_{n_{0}} \tau=-\left(\frac{D_{\tau n_{0}} u}{|\nabla u|}\right) \cdot n .
$$

To compute $D_{n_{0} n_{0} n_{0}} u$, we derive Eq. (1.5) with respect to the field $n_{0}$ :

$$
\alpha D_{n n n_{0}} u+\beta D_{\tau \tau n_{0}} u+\gamma D_{n_{0}}\left(D_{n \tau} u\right)+2 J+I+L=0,
$$

where

$$
\begin{gathered}
J=\alpha\left(D_{\cdot n} u\right) \partial_{n_{0}} n+\beta\left(D_{\cdot \tau} u\right) \partial_{n_{0}} \tau=\frac{\alpha-\beta}{|\nabla u|}\left(D_{n \tau} u\right)\left(D_{n_{0} \tau}\right), \\
I=\gamma\left(\left(D_{n} \cdot u\right) \partial_{n_{0}} n+\left(D_{\tau} \cdot u\right) \partial_{n_{0}} \tau\right)=0, \\
L=\left.\mathcal{F}^{\prime}\right|_{\nabla u \mid} \cdot \partial_{n_{0}}\left(D_{n} u\right)+\mathcal{F}_{u}^{\prime} \cdot \partial_{n_{0}} u .
\end{gathered}
$$

To evaluate these quantities, we use $D_{n \tau} u=0$ on $\partial \Lambda$ and the following

LEMMA 9. - With the above notations, for $h=d(x, \partial \Lambda)>0$ small, then

$$
D_{\tau n_{0}} u=\mathrm{o}(h), \quad|\nabla u| \geqslant C h
$$

for some positive constant $C$, and $D_{n n \tau} u=0$ on $\partial \Lambda$.

As a consequence, on $\partial \Lambda$, we get

$$
\begin{aligned}
& D_{n_{0}}\left(D_{n \tau} u\right)=0, \quad J=0, \quad L=\delta D_{n_{0} n_{0}} u, \\
& \alpha D_{n_{0} n_{0} n_{0}} u+\beta D_{\tau \tau n_{0}} u+\delta D_{n_{0} n_{0}} u=0 .
\end{aligned}
$$

To complete the proof, we have to compute $D_{\tau \tau n_{0}} u$. For that purpose, let us define the function $v(x)=\partial_{n_{0}(x)} u(x)$, which is analytic in a neighbourhood of $\partial \Lambda$ and up to $\partial \Lambda$. It is easy to check that $\partial_{n_{0}} v=D_{n_{0} n_{0}} u$ on $\Omega \backslash \Lambda$. Since $v=0$ and $|\nabla v|=D_{n_{0} n_{0}} u=$ const $>0$ on $\partial \Lambda$, the curvature of $\partial \Lambda \ni x_{0}$ is given by $K\left(x_{0}\right)=\frac{D_{\tau_{0} \tau_{0} v}}{|\nabla v|}$ where $\tau_{0}=-n_{0}^{\perp}$. It is also easy to check that on $\partial \Lambda, D_{\tau_{0} \tau_{0}} v=D_{n_{0} \tau_{0} \tau_{0}} u$. Thus $D_{\tau \tau n_{0}} u=\left(D_{n_{0} n_{0}} u\right) K$ on $\partial \Lambda$, which gives the expected equality and ends the proof of Lemma 8.

Proof of Lemma 9. - Deriving $D_{n n} u=$ constant on $\partial \Lambda$ with respect to $\tau$, we get: $D_{\tau n n} u=0$ on $\partial \Lambda$. Let us remark that

$$
\frac{d}{d h}\left(D_{\tau n} u\left(\gamma_{x_{0}}(h)\right)\right)=D_{\tau n_{0} n_{0}} u-\left(D_{\tau n_{0}} u\right) \frac{D_{n n_{0}} u}{|\nabla u|},
$$

and that $\mid \nabla u\left(\gamma_{x_{0}}(h) \mid \geqslant C h\right.$ because $D_{n_{0} n_{0}} u=$ constant $>0$ on $\partial \Lambda$. Using the regularity of $D^{3} u$, we deduce that $D_{\tau n_{0}} u=\mathrm{o}(h)$ for $h>0$ small, which ends the proof of Lemma 9.

\section{Acknowledgements}

The authors thank H. Berestycki for introducing them to the question of the convexity of the coincidence set for obstacle problems. They also thank B. Teissier for his comments on the properties of the analytic functions and for his suggestions. 


\section{Appendix A}

This last section is devoted to results and extensions that have been omitted in Sections 1-4 to simplify the reading. First, we establish the expression of (1.8) and (1.9) in the case of a ball, which is actually much easier than the general case. The interesting point is that the inequation for the curvature $K$ becomes an equality. Then we give at a formal level the extension of the system (1.8)-(1.9) to dimensions higher than 2 . The estimate for the gradient is unchanged but the one on the curvature is replaced by an estimate on the arithmetic mean curvature, which is not sufficient to prove a convexity result for the free boundary. A rigourous justification of the computations would not be much more difficult than in dimension 2 but is for sure extremely tedious, so we leave it at a formal level. The last part of this appendix is devoted to results on analytic sets that we use in the proof of Theorem 2.

\section{A.1. The radial case}

This method is easy to understand in the radially symmetric case. Assume that $\Omega=B\left(0, R_{0}\right)$ and consider a radial solution of Eq. (1.5). We define for any $t \in\left(0, u_{0}\right)$ the functions $K(t)$ and $m(t)$ by

$$
t=u\left(\frac{1}{K(t)}\right) \quad \text { and } \quad m(t)=u^{\prime}\left(\frac{1}{K(t)}\right) .
$$

A derivation with respect to $t$ gives $\dot{K}=-\frac{K^{2}}{m}$ and $\dot{m}=\frac{u^{\prime \prime}}{m}$. Here $\left({ }^{\circ}\right)$ and ( $)^{\prime}$ respectively denote the derivatives with respect to $t$ and $r$. Eq. (1.5) is equivalent to (1.8). An integration from 0 to $u_{0}$ with the initial values $m(0)=\lambda(K(0))$ and $K\left(u_{0}\right)=\frac{1}{R_{0}}$ gives the result of Theorem 3 . A variant of this approach consists simply to get an upper bound by considering

$$
M\left(u_{0}, K_{0}\right) \geqslant \max _{s \in\left[0, u_{0}\right]} m_{0}(s) \geqslant m(t) \quad \forall t \in\left[0, u_{0}\right], \quad K_{0}=\frac{1}{R_{0}}>0,
$$

where $t \mapsto m_{0}(t)$ is the solution to $\mathcal{F}\left(m_{0} \dot{m_{0}}, m_{0} K_{0}, 0, m_{0}, t\right)=0$ with initial datum $m_{0}(0)=\lambda\left(K_{0}\right)+1$.

\section{A.2. Higher dimensions}

In this subsection, we formally extend our approach to dimensions $d \geqslant 3$. The main difference is that the curvature has to be replaced by the arithmetic mean curvature. We will justify the derivation of this system only at a formal level by considering the generic case.

To simplify the presentation we consider a solution $u$ of

$$
\mathcal{F}\left(D_{d d} u, \sum_{i=1}^{d-1} D_{i i} u,|\nabla u|, u\right)=0
$$


where $D_{i i}$ is defined as follows. Consider the level set $\Gamma^{t}=\{x \in \Omega: u(x)=t\} \subset \mathbb{R}^{d}$ and (when $\nabla u(x) \neq 0$ ) the unit normal vector $n(x)=\frac{\nabla u}{|\nabla u|}(x)$ which is orthogonal to the hyperplane $\Pi=\Pi(x)$ tangent to $\Gamma^{t}$ at $x$. For $i=1,2, \ldots, d-1$, we may diagonalize $\left(D^{2} u\right)_{\Pi}=P_{\Pi}\left(D^{2} u\right) P_{\Pi}$ where $P_{\Pi}$ is the projection on $\Pi$ and define $\tau_{i}$ $(i=1,2, \ldots, d-1)$ as the corresponding eigenvectors such that $\left(\tau_{1}, \tau_{2}, \ldots, \tau_{d-1}, \tau_{d}=\right.$ $n$ ) forms an orthonormal basis in $\mathbb{R}^{d}$ (the derivative along the normal to the level hypersurface, i.e. along the direction $n$, corresponds to the index $d$ ). The numbers $\lambda_{i}=\left(\tau_{i},\left(D^{2} u\right)_{\Pi} \tau_{i}\right)$ are the eigenvalues of $\left(D^{2} u\right)_{\Pi}$ and we define the off-diagonal terms $\mu_{i}=\left(\tau_{d},\left(D^{2} u\right) \tau_{i}\right)=\left(\tau_{i},\left(D^{2} u\right) \tau_{d}\right)$ for $i=1,2, \ldots, d$, and the curvatures $K_{i}=\frac{\lambda_{i}}{|\nabla u|}$ $(i=1,2, \ldots, d-1)$. With the notations $D_{i j} u=\left(\tau_{i},\left(D^{2} u\right) \tau_{j}\right)$, the Fréchet formula are (as in Section $2, \frac{d}{d \tau_{i}}=\tau_{i} \cdot \nabla$, so that two derivatives do not necessarily commute and $\frac{d \tau_{i}}{d \tau_{i}}$ can be different from 0 ):

$$
\begin{aligned}
& \left.\frac{d \tau_{d}}{d \tau_{i}}=\frac{d n}{d \tau_{i}}=\frac{\lambda_{i}}{|\nabla u|} \tau_{i}=K_{i} \tau_{i} \quad \text { (without summation on } i=1,2, \ldots, d-1\right), \\
& \frac{d \tau_{d}}{d \tau_{d}}=\frac{d n}{d n}=\sum_{i=1}^{d-1} \frac{D_{i d} u}{|\nabla u|} \tau_{i},
\end{aligned}
$$

and assuming from now on that $K_{j} \neq K_{i}(1 \leqslant i \neq j \leqslant d-1)$,

$$
\begin{aligned}
& \frac{d \tau_{i}}{d \tau_{d}}=\frac{d \tau_{i}}{d n}=\sum_{\substack{j=1 \\
j \neq i}}^{d} a_{i j} \tau_{j} \quad \text { where } a_{i j}=\frac{2 \frac{\mu_{i} \mu_{j}}{|\nabla u|}-D_{d i j}^{3} u}{|\nabla u|\left(K_{j}-K_{i}\right)}, a_{i d}=-\frac{\mu_{i}}{|\nabla u|}, \\
& \frac{d \tau_{i}}{d \tau_{j}}=\sum_{\substack{k=1 \\
k \neq i}}^{d} a_{i j k} \tau_{k} a_{i j k}=\frac{K_{j}\left(\mu_{i} \delta_{k j}+\mu_{k} \delta_{i j}\right)-D_{i j k} u}{|\nabla u|\left(K_{k}-K_{i}\right)}, \quad a_{i j d}=-K_{j} \delta_{i j} \\
& \text { for } 1 \leqslant k \neq i \leqslant d-1 .
\end{aligned}
$$

As in Section 2, we denote by $x^{t} \in \Gamma^{t}$ a point which realizes the maximum of $|\nabla u|^{2}$ on $\Gamma^{t}$ and assume that $t \mapsto x^{t}$ is an analytic curve. By definition of $x^{t}, \frac{d}{d \tau_{i}}\left(|\nabla u|^{2}\right)\left(x^{t}\right)=$ $0(i=1,2, \ldots, d-1)$, thus proving that $\left.\mu_{i}\right|_{x=x^{t}}=0$. Because $|\nabla u|^{2}$ restricted to $\Gamma^{t}$ has a critical point at $x=x^{t}$, we may also define its Hessian as

$$
\frac{d}{d \tau_{i}}\left(\frac{d}{d \tau_{j}}\left(|\nabla u|^{2}\right)\right)=\frac{d}{d \tau_{j}}\left(\frac{d}{d \tau_{i}}\left(|\nabla u|^{2}\right)\right)=: H\left(\tau_{i}, \tau_{j}\right) \leqslant 0,
$$

with

$$
\begin{aligned}
& H=\left(D^{2}\left(|\nabla u|^{2}\right)\right)_{\Pi}-2\left(D_{d d} u\right)\left(D^{2} u\right)_{\Pi} \quad \text { and } \\
& \left(D^{2}\left(|\nabla u|^{2}\right)\right)_{\Pi}=2\left(\left(D^{2} u\right)_{\Pi}\right)^{2}+2|\nabla u|\left(D_{d . .} u\right)_{\Pi} .
\end{aligned}
$$

In the following, we shall assume for simplicity that $H$ is actually negative definite. Let us compute $\frac{d}{d t}\left(\sum_{i=1}^{d-1} K_{i}\right)$. 
(1) With notations similar to the ones of the 2-dimensional case, we have

$$
\frac{1}{\delta t}\left(x^{t+\delta t}-x^{t}\right)=\left(\frac{1}{|\nabla u|}+b \delta t\right) n+(\vec{h}+\vec{B} \delta t)+\mathrm{O}\left((\delta t)^{2}\right)
$$

where $\vec{h}=\left(h_{1}, h_{2}, \ldots, h_{d-1}\right), \vec{B} \in \Pi\left(x^{t}\right)$. With $\vec{\delta}=x^{t+\delta t}-x^{t}$,

$$
\begin{aligned}
\delta t=u\left(x^{t+\delta t}\right)-u\left(x^{t}\right) & =\vec{\delta} \cdot \nabla u+\frac{1}{2}\left(\vec{\delta} \cdot\left(D^{2} u\right) \vec{\delta}\right)+\mathrm{o}\left(|\vec{\delta}|^{2}\right) \\
& =\delta t+\left(b|\nabla u|+\frac{1}{2}\left(\vec{h} \cdot\left(D^{2} u\right) \vec{h}\right)+\frac{1}{2} \frac{D_{d d} u}{|\nabla u|^{2}}\right)(\delta t)^{2}+\mathrm{o}\left((\delta t)^{2}\right), \\
& \text { and } \quad b=-\frac{1}{2}\left(\vec{h} \cdot\left(D^{2} u\right)_{\Pi} \vec{h}\right)-\frac{1}{2} \frac{D_{d d} u}{|\nabla u|^{3}}
\end{aligned}
$$

(2) Using the Taylor expansion of $\left|\nabla u\left(x^{t+\delta t}\right)\right|^{2}-\left|\nabla u\left(x^{t}\right)\right|^{2}$ and maximizing it with respect to $\vec{h}$, we get $\vec{h}=-\frac{1}{|\nabla u|}\left(H^{(-1)} \circ P_{\Pi}\right)\left(D_{d} \cdot\left(|\nabla u|^{2}\right)\right)$. Since $D_{d j}\left(|\nabla u|^{2}\right)=$ $2|\nabla u| D_{d d j} u$, we have

$$
\vec{h}=-2\left(H^{(-1)} \circ P_{\Pi}\right)\left(D_{d d} \cdot u\right) .
$$

(3) We compute $\sum_{i=1}^{d-1} \dot{K}_{i}$ :

$$
\sum_{i=1}^{d-1} \dot{K}_{i}=\frac{d}{d t}\left(\sum_{i=1}^{d-1} K_{i}\left(x^{t}\right)\right)=\frac{1}{|\nabla u|} \frac{d}{d n}\left(\sum_{i=1}^{d-1} K_{i}\right)+\vec{h} \cdot \nabla_{\Pi}\left(\sum_{i=1}^{d-1} K_{i}\right) .
$$

Using the Fréchet formulas, we get, at $x=x^{t}$,

$$
\frac{d K_{i}}{d n}=\frac{1}{|\nabla u|}\left(D_{d i i} u-D_{d d} u K_{i}\right) \quad \text { and } \quad \frac{d K_{i}}{d \tau_{j}}=\frac{1}{|\nabla u|} D_{i i j} u .
$$

(4) On one hand, let us remark that because $H<0$, for each $i=1, \ldots, d-1$ :

$$
0 \geqslant H_{i i}=2|\nabla u|\left(D_{d i i} u+|\nabla u| K_{i}^{2}-D_{d d} u K_{i}\right)
$$

So that $\frac{1}{|\nabla u|} \frac{d K_{i}}{d n} \leqslant-\frac{K_{i}^{2}}{|\nabla u|}$.

(5) On the other hand, deriving Eq. (A.1) with respect to $\tau_{j}$, we obtain $\alpha D_{d d j} u+$ $\beta \sum_{i} D_{i i j} u=0$, and consequently $\frac{d}{d \tau_{j}}\left(\sum_{i=1}^{d-1} K_{i}\right)=-\frac{\alpha}{\beta} \frac{D_{d d j} u}{|\nabla u|}$ and

$$
\vec{h} \cdot \nabla_{\Pi}\left(\sum_{i=1}^{d-1} K_{i}\right)=2 \frac{\alpha}{\beta}\left[P_{\Pi}\left(D_{d d} \cdot u\right)\right] H^{(-1)}\left[P_{\Pi}\left(D_{d d} \cdot u\right)\right] \leqslant 0
$$

because $H<0$. Therefore

$$
\frac{d}{d t}\left(\sum_{i=1}^{d-1} K_{i}\right) \leqslant-\frac{1}{|\nabla u|} \sum_{i=1}^{d-1} K_{i}^{2}
$$


In view of the free boundary problem, we may simply quote that if the domain $\Omega \subset \mathbb{R}^{d}$ is convex, the mean curvature $\sum_{i=1}^{d-1} K_{i}$ of the free boundary at the limit of the points that maximize the gradient, is positive. Concerning the estimates on the gradient, Theorem 2 could be generalized to any dimension, thus providing an estimate taking the geometry of the domain into account. However a rigourous justification of these estimates would involve a tedious discussion of the various special cases (that we discarded above by taking appropriate assumptions), similar for the methods to the 2-dimensional case, but much longer. This is why we left it here at a formal level.

\section{A.3. Results on analytic sets}

We will first state some general results on analytic sets and then prove Lemma 5 and Lemma 7.

From [9] (Chapter 8: Etude locale des fonctions et des ensembles analytiques; Propositions 4.2.5, 7.2, 7.7 and Theorem 1.2.2) we deduce the

THEOREM 4. - For $N \geqslant 1$, let $U$ be an open set of $\mathbb{R}^{2}$ and $F_{i}\left(x_{1}, x_{2}\right), i=1,2, \ldots, N$, be real analytic functions of $\left(x_{1}, x_{2}\right) \in U$. We assume that $F_{1} \not \equiv 0$ and $F_{i}(0)=0$, $i=1,2, \ldots, N$. Then there exists positive real number $r$ and an integer $k$ such that

$$
\left(\bigcap_{i=1}^{N}\left\{F_{i}=0\right\}\right) \cap B_{r}(0)=\{0\} \cup\left(\bigcup_{j=1}^{k} \gamma_{j}\right)
$$

for a disjoint union of analytic open curves $s \mapsto \gamma_{j}(s), s \in(0,1)$, with

$$
\left\{\begin{array}{l}
\lim _{s \rightarrow 0} \gamma_{j}(s)=0, \\
\lim _{s \rightarrow 1} \gamma_{j}(s)=x_{j} \in \partial B_{r}(0) .
\end{array}\right.
$$

Moreover the same property is true for every ball $B_{r^{\prime}}(0)$ with $r^{\prime}<r$.

This result gives a precise description of the structure of analytic sets. In our proof of Theorem 2, we are interested in the following special situation. Let $F_{1} \not \equiv 0$ be an analytic function with $F_{1}(0)=0$. Theorem 4 for $N=1$ gives the existence of an open curve $\gamma \subset\left\{F_{1}=0\right\}$ with $\lim _{s \rightarrow 0} \gamma(s)=0$. Let $F_{0}$ be a second analytic function such that $F_{0}(0)=0$ and $\nabla F_{0}(0) \neq 0$. What can be said on $\frac{d}{d s}\left(F_{0} \circ \gamma\right)$ ? The answer to this question is given by the

COROLlaRY 1. - Consider a real analytic function $F_{0}$ of the variables $\left(x_{1}, x_{2}\right) \in U$, where $U$ is an open set in $\mathbb{R}^{2}$, such that $F_{0}(0)=0$ and $\nabla F_{0}(0) \neq 0$. If $\gamma:(0,1) \rightarrow U$ is an analytic curve such that $\lim _{s \rightarrow 0} \gamma(s)=0$ and $\gamma \subset\left\{F_{1}=0\right\}$, where the function $F_{1}$ is analytic with $F_{1} \not \equiv 0$, then for an $\varepsilon>0$ small enough, on the interval $(0, \varepsilon)$,

(i) either $\frac{d}{d s}\left(F_{0} \circ \gamma\right) \equiv 0$,

(ii) or $\pm \frac{d}{d s}\left(F_{0} \circ \gamma\right)>0$.

The proof of this corollary takes advantage of the following classical result. 
PROPOSITION 4. - Let $g$ and $h$ be two analytic functions defined on the interval $(-1,1)$. If 0 is an accumulation point of the set $\{s \in(-1,1): f(s)=g(s)\}$, then $f \equiv g$ on $(-1,1)$.

Proof of Corollary 1. - Consider an analytic function $F_{1}$ defined on $U$ such that $\gamma \subset\left\{F_{1}=0\right\}$. Let $l$ be the smallest integer such that $\gamma \subset \bigcap_{j=0}^{l}\left\{D^{j} F_{1}=0\right\}$ and $\gamma \not \subset\left\{D^{l+1} F_{1}=0\right\}$, where $D^{j} F_{1}$ denotes the set of all partial derivatives of total order $j:\left\{\partial_{1}^{j_{1}} \partial_{2}^{j_{2}} F_{1}\right\}_{j_{1}+j_{2}=j}$. We know that $F_{1} \not \equiv 0$, so $l$ is finite and there exists $j_{1}, j_{2} \geqslant 0$, $j_{1}+j_{2}=l$ such that for $\tilde{F}_{1}=\partial_{1}^{j_{1}} \partial_{2}^{j_{2}} F_{1}$ we have

$$
\tilde{F}_{1} \circ \gamma \equiv 0 \quad \text { and } \quad\left(\nabla \tilde{F}_{1}\right) \circ \gamma \not \equiv 0 .
$$

Let $\tau$ be the unit vector field tangent to level lines of $F_{0}$ (i.e. such that $\partial_{\tau} F_{0}=0$ ).

Case 1: $\tau \cdot \nabla \tilde{F}_{1}(0) \neq 0$ : the curve $\gamma$ is analytic in a neighbourhood of 0 and up to $s=0$. In particular we can chose the curvilinear abscissa $s$ as a parametrization up to $s=0$ and

$$
\frac{d}{d s}\left(F_{0} \circ \gamma\right)=\frac{d \gamma}{d s} \cdot \nabla F_{0}=-\left|\nabla F_{0}\right|\left(\frac{d \gamma}{d s}\right)^{\perp} \cdot \tau
$$

because $\tau=-\frac{\left(\nabla F_{0}\right)^{\perp}}{\left|\nabla F_{0}\right|}$. We know that $\left(\frac{d \gamma}{d s}\right)^{\perp}$ is colinear to $\nabla \tilde{F}_{1}$, and consequently

$$
\frac{d}{d s}\left(F_{0} \circ \gamma\right)= \pm \frac{\left|\nabla F_{0}\right|}{\left|\nabla \tilde{F}_{1}\right|}\left(\tau \cdot \nabla \tilde{F}_{1}\right)
$$

so we deduce that $\pm \frac{d}{d s}\left(F_{0} \circ \gamma\right)>0$ in a neighbourhood of 0 .

Case 2: $\tau \cdot \nabla \tilde{F}_{1}(0)=0$ : if $\tau \cdot \nabla \tilde{F}_{1} \equiv 0$ on $U$, then obviously $\left(\tau \cdot \nabla \tilde{F}_{1}\right) \circ \gamma \equiv 0$ and $\frac{d}{d s}\left(F_{0} \circ \gamma\right) \equiv 0$. If $\tau \cdot \nabla \tilde{F}_{1} \not \equiv 0$ on $U$, then from Theorem 4 we have

$$
\left(\left\{\tilde{F}_{1}=0\right\} \cap\left\{\tau \cdot \nabla \tilde{F}_{1}=0\right\}\right) \cap B_{r}(0)=\{0\} \cup\left(\bigcup_{j=1}^{k} \gamma_{j}\right)
$$

for some $r>0$ small enough. In that case, either for any $j, \gamma_{j} \neq \gamma$ and then $\pm(\tau$. $\left.\nabla \tilde{F}_{1}\right)\left.\right|_{\gamma}>0$ : as in Case 1, Eq. (A.3), we get $\pm \frac{d}{d s}\left(F_{0} \circ \gamma\right)>0$ in a neighbourhood of 0 , or the exists some $j$ such that $\gamma_{j}=\gamma$. In that case, $\tau \cdot \nabla \tilde{F}_{1} \circ \gamma \equiv 0$ on a neighborhood of 0 . From (A.2) we know that $\left(\nabla \tilde{F}_{1} \circ \gamma\right)(s) \neq 0$ except maybe in a decreasing sequence of points $\left(s_{n}\right)_{n \in \mathbb{N}} \in(0,1)^{\mathbb{N}}$. Because the map $(0,1) \ni s \mapsto\left(\nabla \tilde{F}_{1} \circ \gamma\right)(s)$ is analytic, the only possible accumulation point of the sequence $\left(s_{n}\right)_{n \in \mathbb{N}}$ is 0 according to Proposition 4 . Away from these points $s_{n}$, we can apply the implicit function theorem which proves that $F_{0} \circ \gamma=\mathrm{const}=C_{n}$ on $\left(s_{n+1}, s_{n}\right)$. By continuity at $s_{n}$, we get $C_{n}=C_{n+1}=F_{0}(0)=0$ and consequently $\gamma \subset\left\{F_{0}=0\right\}, \frac{d}{d s}\left(F_{0} \circ \gamma\right) \equiv 0$ on $(0, \varepsilon)$ for $\varepsilon>0$ small enough.

We are now going to prove Lemma 5 and Lemma 7 which are used in the proof of Theorem 2.

Proof of Lemma 5. - The map $t \mapsto m(t)$ is continuous on $\left[0, u_{0}\right]$. For every $0<\varepsilon^{\prime}<\varepsilon$ we have $\overline{\omega_{\varepsilon}} \subset \omega_{\varepsilon^{\prime}}$. Let $F_{\omega_{\varepsilon}}=\{F=0\} \cap \omega_{\varepsilon}$. Then $\overline{F_{\omega_{\varepsilon}}}$ is a compact set included in 
$\{|\nabla u|>0\}$. At every point $x_{0} \in \overline{F_{\omega_{\varepsilon}}}$, the set $\{F=0\}$ has the property given in Theorem 4: for any $x_{0} \in \overline{F_{\omega_{\varepsilon}}}$ there exists an $r_{x_{0}}>0$, and a finite set of curves $\left(\gamma_{j}^{x_{0}}\right)_{j=1}^{k_{x_{0}}}$ such that

$$
\{F=0\} \cap B_{r_{0}}\left(x_{0}\right)=\left\{x_{0}\right\} \cup\left(\bigcup_{j=1}^{k_{x_{0}}} \gamma_{j}^{x_{0}}\right) .
$$

Because $\overline{F_{\omega_{\varepsilon}}}$ is compact, it can be covered by a finite number, $N$, of balls

$$
\overline{F_{\omega_{\varepsilon}}} \subset \bigcup_{i=1}^{N} B_{r_{i}}\left(x_{i}\right)=\omega,
$$

where $r_{i}=\frac{r_{x_{i}}}{2}$. The result of Lemma 5 is then a straightforward consequence of Corollary 1.

Proof of Lemma 7. - To prove (3.6), we now consider a point $x_{0} \in X^{t_{k}^{*}}$ such that $K\left(x_{0}\right)=\inf _{y \in X^{t^{*}} k} K(y)$. We will prove that

$$
\limsup _{\delta \rightarrow 0^{+}} K\left(t_{k}^{*}+\delta\right) \leqslant K\left(x_{0}\right) .
$$

To this end, let us consider a smooth curve $\gamma_{0}$ defined for $t \in\left(t_{k}^{*}-\eta, t_{k}^{*}+\eta\right)$ for some small $\eta>0$, such that $\gamma_{0}\left(t_{k}^{*}\right)=x_{0}$ and $u\left(\gamma_{0}(t)\right)=t$. Then by definition of $m(t)$, we have

$$
\frac{1}{\delta}\left(m\left(t_{k}^{*}+\delta\right)-m\left(t_{k}^{*}\right)\right) \geqslant \frac{1}{\delta}\left(\left|\nabla u\left(\gamma_{0}\left(t_{k}^{*}+\delta\right)\right)\right|-\left|\nabla u\left(\gamma_{0}\left(t_{k}^{*}\right)\right)\right|\right) \quad \text { for } \delta \geqslant 0 .
$$

Because of Assumption (A2), equation $\mathcal{F}\left(D_{n n} u, D_{\tau \tau} u, D_{n \tau} u,|\nabla u|, u\right)=0$ can now be rewritten locally near $x_{0}$ as

$$
D_{n n} u=\mathcal{H}\left(D_{\tau \tau} u, D_{n \tau} u,|\nabla u|, u\right)
$$

where $\mathcal{H}$ is analytic in all the variables and (locally) decreasing in $D_{\tau \tau} u$. With the notation $m_{0}(t)=\left|\nabla u\left(\gamma_{0}(t)\right)\right|$,

$$
\begin{aligned}
\lim _{\delta \rightarrow 0^{+}} & \frac{1}{\delta}\left(\left|\nabla u\left(\gamma_{0}\left(t_{k}^{*}+\delta\right)\right)\right|-\left|\nabla u\left(\gamma_{0}\left(t_{k}^{*}\right)\right)\right|\right) \\
= & \lim _{\delta \rightarrow 0^{+}} \frac{1}{\delta} \int_{t_{k}^{*}}^{t_{k}^{*}+\delta} \frac{1}{m_{0}} \mathcal{H}\left(m_{0} K\left(\gamma_{0}(t)\right), D_{n \tau} u\left(\gamma_{0}(t)\right), m_{0}, t\right) d t \\
\quad= & \frac{1}{m} \mathcal{H}\left(m K\left(x_{0}\right), 0, m, t_{k}^{*}\right) \quad \text { for } m=m\left(t_{k}^{*}\right),
\end{aligned}
$$

by continuity of all the quantities on the smooth curve $\gamma_{0}$. Let us recall that $m(t)=$ $\sup _{\gamma \in \mathcal{G}_{k}}|\nabla u(\gamma(t))|$ for $t \in\left(t_{k}^{*}, t_{k+1}^{*}\right)$. Because we restrict our study the case $t>t_{k}^{*}$, close to $t_{k}^{*}$, we only need to consider

$$
\mathcal{F}_{k}^{*}:=\left\{\gamma \in \mathcal{F}_{k}:\left|\nabla u\left(\gamma\left(t_{k}^{*}\right)\right)\right|=m\left(t_{k}^{*}\right)\right\} .
$$


Then locally for $t>t_{k}^{*}$, we have $m(t)=\sup _{\gamma \in \mathcal{G}_{k}^{*}}|\nabla u(\gamma(t))|$ and for each curve $\gamma \in \mathcal{F}_{k}^{*}$ we get similarly:

$$
\lim _{\delta \rightarrow 0^{+}} \frac{1}{\delta}\left(\left|\nabla u\left(\gamma\left(t_{k}^{*}+\delta\right)\right)\right|-\left|\nabla u\left(\gamma\left(t_{k}^{*}\right)\right)\right|\right)=\frac{1}{m} \mathcal{H}\left(m K\left(\gamma\left(t_{k}^{*}\right)\right), 0, m, t\right)
$$

for $m=m\left(t_{k}^{*}\right)$. Because

$$
\frac{1}{\delta}\left(m\left(t_{k}^{*}+\delta\right)-m\left(t_{k}^{*}\right)\right)=\sup _{\gamma \in \mathcal{G}_{k}^{*}} \frac{1}{\delta}\left(\left|\nabla\left(\gamma\left(t_{k}^{*}+\delta\right)\right)\right|-\left|\nabla u\left(\gamma\left(t_{k}^{*}\right)\right)\right|\right)
$$

and because $\mathcal{G}_{k}^{*}$ is finite, we have: $\lim _{\delta} \sup _{\mathcal{G}_{k}^{*}}=\sup _{\mathcal{G}_{k}^{*}} \lim _{\delta}$, which implies

$$
\begin{aligned}
\lim _{\delta \rightarrow 0^{+}} & \frac{1}{\delta}\left(m\left(t_{k}^{*}+\delta\right)-m\left(t_{k}^{*}\right)\right) \\
= & \frac{1}{m} \mathcal{H}\left(m, \inf _{\gamma \in \mathcal{G}_{k}^{*}} K\left(\gamma\left(t_{k}^{*}\right)\right), 0, m, t_{k}^{*}\right) \quad \text { for } m=m\left(t_{k}^{*}\right) .
\end{aligned}
$$

From (A.5) we deduce

$$
\mathcal{H}\left(m \inf _{\gamma \in \mathcal{G}_{k}^{*}} K\left(\gamma\left(t_{k}^{*}+\delta\right)\right), 0, m, t_{k}^{*}\right) \geqslant \mathcal{H}\left(m K\left(x_{0}\right), 0, m, t_{k}^{*}\right),
$$

which gives $\inf _{\gamma \in \mathcal{G}_{k}^{*}} K\left(\gamma\left(t_{k}^{*}\right)\right) \leqslant K\left(x_{0}\right)$. To conclude, we remark that

$$
\begin{aligned}
\limsup _{\delta \rightarrow 0^{+}} K\left(t_{k}^{*}+\delta\right) & =\limsup _{t \rightarrow\left(t_{k}^{*}\right)^{+}}\left(\inf _{\gamma \in \mathcal{G}_{k}^{*},|\nabla u(\gamma(t))|=m(t)} K(\gamma(t))\right) \\
& \leqslant \lim _{t \rightarrow\left(t_{k}^{*}\right)^{+}}\left(\inf _{\gamma \in \mathcal{G}_{k}^{*}} K(\gamma(t))\right) \\
& =\inf _{\gamma \in \mathcal{G}_{k}^{*}}\left(\lim _{t \rightarrow\left(t_{k}^{*}\right)^{+}} K(\gamma(t))\right) \\
& =\inf _{\gamma \in \mathcal{G}_{k}^{*}} K\left(\gamma\left(t_{k}^{*}\right)\right) \leqslant K\left(x_{0}\right),
\end{aligned}
$$

which is nothing else than (A.4). Similarly we get $\liminf _{\delta \rightarrow 0^{+}} K\left(t_{k}^{*}-\delta\right) \geqslant K\left(x_{0}\right)$, which with (A.4) implies (3.6). This ends the proof of Lemma 7.

\section{REFERENCES}

[1] H.W. Alt, D. Phillips, A free boundary problem for semilinear elliptic equations, J. Reine Angew. Math. 368 (1986) 63-107.

[2] A. Bonnet, R. Monneau, Distribution of vortices in a type II superconductor as a free boundary problem: Existence and regularity via Nash-Moser theory, Interfaces and Free Boundaries 2 (2000) 181-200.

[3] H. Brézis, D. Kinderlehrer, The smoothness of solutions to nonlinear variational inequalities, Indiana Univ. Math. J. 23 (9) (1974) 831-844.

[4] L.A. Caffarelli, Compactness method in free boundary problems, Comm. P.D.E. 5 (4) (1980) 427-448. 
[5] L.A. Caffarelli, N.M. Rivière, Smoothness and analyticity of free boundaries in variational inequalities, Ann. Scuola Norm. Sup. Pisa, serie IV 3 (1975) 289-310.

[6] L.A. Caffarelli, J. Salazar, Solutions of fully nonlinear elliptic equations with patches of zero gradient: existence, regularity and convexity of level curves, Trans. Amer. Math. Soc. 354 (8) (2002) 3095-3115.

[7] L.A. Caffarelli, J. Spruck, Convexity properties of solutions to some classical variational problems, Comm. P.D.E. 7 (11) (1982) 1337-1379.

[8] J.I. Diaz, B. Kawohl, On convexity and starshapedness of level sets for some nonlinear elliptic and parabolic problems on convex rings, J. Math. Anal. Appl. 177 (1993) 263-286.

[9] P. Dolbeault, Analyse Complexe, in: Collection Maîtrise de Mathematiques Pures, Masson, 1990.

[10] J. Dolbeault, R. Monneau, Estimations de convexité pour des équations elliptiques nonlinéaires et application à des problèmes de frontière libre [Convexity estimates for nonlinear elliptic equations and application to free boundary problems], C. R. Acad. Sci. Paris Sér. I 331 (2000) 771-776.

[11] J. Frehse, On the regularity of the solution of a second order variational inequality, Boll. U.M.I. 6 (4) (1972) 312-315.

[12] A. Friedman, Variational Principles and Free Boundary Problems, in: Pure and Applied Mathematics, Wiley-Interscience, 1982.

[13] A. Friedman, D. Phillips, The free boundary of a semilinear elliptic equation, Trans. Amer. Math. Soc. 282 (1984) 153-182.

[14] R.S. Hamilton, The inverse function theorem of Nash and Moser, Bull. Amer. Math. Soc. 7 (1982) 65-222.

[15] A. Henrot, H. Shahgholian, Convexity of free boundaries with Bernoulli type boundary conditions, Nonlinear Analysis T.M.A. 28 (5) (1997).

[16] A. Henrot, H. Shahgholian, Existence of classical solutions to a free boundary problem for the p-Laplace operator: (I) the exterior convex case, J. Reine Angew. Math. 521 (2000) 85-97.

[17] A. Henrot, H. Shahgholian, Existence of classical solutions to a free boundary problem for the p-Laplace operator: (II) the interior convex case, Indiana Univ. Math. J. 49 (1) (2000) 311-323.

[18] B. Kaup, L. Kaup, Holomorphic Functions of Several Variables, Walter de Gruyter, Berlin, 1983.

[19] B. Kawohl, When are solutions to nonlinear elliptic boundary value problems convex?, Comm. P.D.E. 10 (1985) 1213-1225.

[20] B. Kawohl, Rearrangements and Convexity of Level Sets in PDE, in: Lecture Notes in Math., Vol. 1150, Springer, 1985.

[21] B. Kawohl, On the convexity and symmetry of solutions to an elliptic free boundary problem, J. Austral. Math. Soc. (Series A) 42 (1987) 57-68.

[22] B. Kawohl, On the convexity of level sets for elliptic and parabolic exterior boundary value problems, in: Potential Theory, Prague, 1987, Plenum, New York, 1988, pp. 153-159.

[23] D. Kinderlehrer, L. Nirenberg, Regularity in free boundary problems, Bull. Amer. Math. Soc. 7 (1982) 65-222.

[24] D. Kinderlehrer, G. Stampacchia, An Introduction to Variational Inequalities and Their Applications, Academic Press, New York, 1980.

[25] P. Laurence, E. Stredulinsky, Existence of regular solutions with levels for semilinear elliptic equations with nonmonotone $L^{1}$ nonlinearities, Indiana Univ. Math. J. 39 (4) (1990) 10811114. 
[26] R. Monneau, Problèmes de frontières libres, EDP elliptiques non linéaires et applications en combustion, supraconductivité et élasticité, Thèse de doctorat de l'Université de Paris VI, 1999.

[27] C.B. Morrey, Multiple Integrals in the Calculus of Variations, in: Die Grundlehren der Mathematischen Wissenschaften in Einzeldarstellungen, Vol. 130, Springer-Verlag, New York, 1966.

[28] J.F. Rodrigues, Obstacle Problems in Mathematical Physics, North-Holland, Amsterdam, 1987.

[29] D.G. Schaeffer, One-sided estimates for the curvature of the free boundary in the obstacle problem, Adv. in Math. 24 (1977) 78-98.

[30] G. Talenti, Some estimates of solutions to Monge-Ampère type equations in dimension two, Ann. Scuola Norm. Sup. Pisa Cl. Sci., IV. Ser. 8 (1981) 183-230. 